\title{
EFFECT OF DIETARY PROTECTED FAT AND ROUGHAGE LEVEL ON DIGESTION, RUMEN METABOLISM AND PLASMA LIPIDS OF GROWING-FINISHING LAMBS
}

\author{
T.M. El-Bedawy ${ }^{1}$, Sawsan M. Ahmed ${ }^{2}$, M.A.I. Salem ${ }^{1}$ and H. A.A. Omer ${ }^{2}$
}

1- Department of Animal Production, Faculty of Agriculture, Cairo University, Giza, Egypt, 2- Department of Animal Production, National Research Center, Dokki, Cairo, Egypt

\section{SUMMARY}

Sixty male Barki lambs, 8 month old and $27.5 \mathrm{~kg}$ average body weight, were randomly divided into six similar groups. Lambs were fed ration at level of $3.5 \%$ of body weight for 70 days. Rations were iso nitrogenous containing $10 \%$ or $30 \%$ berseem hay each with 0, 4 or $8 \%$ Ca-Soap.

At the end of growth trail, twenty-four metabolism trials were conducted to evaluate nutrient digestibilities, nutritive value of the experimental rations, nitrogen balance and water intakes. Ruminal fluid samples were collected at 0 and 4 hrs post feeding to determine $\mathrm{pH}$, ammonia-nitrogen, total VFA'S concentrations and molar proportions of fatty acids, total nitrogen and non- protein nitrogen. Blood plasma total lipids, triglycerides and cholesterol were also determined.

Results indicated feeding $4 \%$ calcium soap rations $(P<0.05)$ improved nutrient digestibilities but this effect was not evident by feeding with $8 \%$ calcium soap supplement. Increasing roughage from $10 \%$ to $30 \%$ had no significant effect on nutrient digestibility. Cell wall constituents digestibilities were higher for $30 \%$ roughage rations and positively affected by fat supplement. Higher energetic values (DE and TDN) was associated with fat supplement and low roughage levels. The DCP values were not affect by the dietary treatments.

Calcium soap supplement significantly improved DE and TDN intakes specially with high roughage level but had no significant effect on DCP intake. Water intake $(P<0.05)$ increased by increasing fat supplement to $8 \%$ and roughage level to $30 \%$. Nitrogen balance was not affected by the dietary treatments.

Fat Supplement had no significant effect on ruminal parameters. However, increasing roughage level increased $(P<0.05)$ ruminal $\mathrm{pH}$, acetate concentration and acetate: propionate ratio but decreased propionate, iso butyrate and valerate. Ruminal total VFA's concentrations, butyrate and iso valerate were not affected by dietary roughage level. Lower $\mathrm{pH}$ and higher total VFA's concentrations, acetate, butyrate, iso butyrate, valerate and iso valerate were found at $4 \mathrm{hrs}$ post-feeding, but propionate and acetate: propionate ratio were not affected by sampling time. Increasing calcium soap level in sheep diet had no significant effect on rumen nitrogen metabolism. However, increasing roughage level $(P<0.05)$ decreased ruminal total nitrogen, non protein nitrogen ammonia nitrogen and true protein nitrogen. All forms of ruminal nitrogen were $(P<0.05)$ lower at 4 hrs post feeding than those before feeding

Increasing protected fat level to $4 \%$ or $8 \%$ of ration dry matter significantly increased plasma total lipids and $(P<0.05)$ triglycerides. Total cholesterol was 
$(P<0.05)$ higher for $8 \%$ fat group than the control and $4 \%$ fat group but increasing roughage level $(P<0.05)$ increased total plasma lipids and total cholesterol but had no significant effect on triglycerides. Total cholesterol $(P<0.05)$, total lipids and triglycerides $(P>0.05)$ were higher at 4 hrs post feeding than those before feeding.

Inclusion of $4 \%$ calcium soap improved the nutrient utilization of high $(30 \%)$ roughage diet to be comparable to that of low (10\%) roughage one with no adverse effect on rumen metabolism or blood plasma metabolites.

Keywords: sheep, calcium soaps, digestion, rumen metabolism, plasma lipids

\section{INTRODUCTION}

Fat can be used to increase the energy density of high-forage diets fed to ruminants. However, it is believed that lipid supplementation to ruminant diets often has a negative effect on feed intake and fiber digestibility (Corine et al., 1993). This effect is more marked with polyunsaturated fatty acids (Palmquist and Jenkins, 1980; Sutton et al., 1983). Lower digestibility of fiber may reduce the ratio of acetic to propionic acids in the rumen and also may decrease digestible energy (Chalupa et al., 1984; Broudiscou et al., 1988).

Because only 3 to $5 \%$ of fat added to common feeds seems to be tolerated by ruminal microorganisms (Palmquist and Jenkins, 1980), research has been conducted to develop high- fat feeds that do not impair fermentative digestion i.e. encapsulated fat, prilled fat, and calcium salt of fatty acids (Grummer, 1988). The use of these fats suggests the potential to employ lipids up to 8 to $9 \%$ of the diet DM (Ostergaard et al., 1981).

Protected fats are widely used in dairy rations, less for growing-finishing steer and not often in growing sheep diets. The objective of this work is to study the effects of supplemental protected fat on lamb performance, nutrient digestibility, feed intake, rumen function and blood lipid metabolites of lambs fed low or high roughage diets.

\section{MATERIALS AND METHODS}

Sixty male Barki lambs aged 8 months with $27.5 \mathrm{~kg}$ average body weight were randomly divided into six similar groups, each of ten lambs and adapted to the experimental rations for one week before data collection.

Lambs were fed at DM Level of $3.5 \%$ body weight for 70 days. Six diets containing $10 \%$ or $30 \%$ berseem hay as roughage source and $\mathrm{Ca}$-Soaps with three levels 0 (low), 4 (medium) and $8 \%$ (high) of dietary DM. Ingredient and chemical composition of the experimental rations are presented in Table 1.

\section{Metabolism trial}

Six metabolism trial were conducted to determine the nutrient digestibilities and nutritive value of the experimental rations. Nitrogen balance and water intakes were also determined. Four lambs from each group were housed in metabolic cages for 8 days as a preliminary period followed by 7 days for total collection. The experimental rations were offered once a day at 09:30 hrs. Feces and urine were collected before feeding at 08:30. Actual feed intake, fresh weight of feces, urine volume and water intake were daily recorded. Chemical composition of rations, feces 
and urine was carried out according to the standard methods of A.O.A.C. (1984). Cell wall constituents were determined in feeds and feces according to Goering and Van Soest (1970).

Table 1. Feed and chemical composition of the experimental rations

\begin{tabular}{|c|c|c|c|c|c|c|}
\hline \multirow[t]{2}{*}{ Item } & \multicolumn{3}{|c|}{$\begin{array}{c}10 \% \text { roughage } \\
\text { Calcium soap, } \%\end{array}$} & \multicolumn{3}{|c|}{$\begin{array}{c}30 \% \text { roughage } \\
\text { Calcium soap, \% }\end{array}$} \\
\hline & 0 & 4 & 8 & 0 & 4 & 8 \\
\hline \multicolumn{7}{|c|}{ Feed composition, \% } \\
\hline Concentrate mix. ${ }^{1}$ & 89.7 & 85.2 & 81.7 & 73.5 & 70.2 & 66.9 \\
\hline Berseem hay & 10.3 & 10.6 & 10.3 & 26.5 & 25.6 & 25.1 \\
\hline Protected fat ${ }^{2}$ & 0 & 4.2 & 8.0 & 0 & 4.2 & 8.0 \\
\hline \multicolumn{7}{|c|}{ Chemical composition, \% } \\
\hline DM & 89.32 & 89.52 & 89.48 & 90.68 & 91.06 & 91.45 \\
\hline \multicolumn{7}{|l|}{ DM composition\% } \\
\hline OM & 94.18 & 93.62 & 93.04 & 93.20 & 92.66 & 92.14 \\
\hline $\mathrm{CP}$ & 19.38 & 19.07 & 18.81 & 19.09 & 18.75 & 17.35 \\
\hline $\mathrm{EE}$ & 2.90 & 6.37 & 9.84 & 2.65 & 6.12 & 9.59 \\
\hline $\mathrm{CF}$ & 5.22 & 5.27 & 5.16 & 9.65 & 9.61 & 9.91 \\
\hline NFE & 66.68 & 62.91 & 59.23 & 61.81 & 58.18 & 55.29 \\
\hline Ash & 5.82 & 6.38 & 6.96 & 6.80 & 7.34 & 7.86 \\
\hline \multicolumn{7}{|c|}{ Cell wall constituents, \% } \\
\hline NDF & 19.41 & 18.99 & 18.78 & 31.64 & 31.21 & 30.56 \\
\hline ADF & 5.97 & 6.35 & 5.80 & 11.80 & 12.19 & 12.56 \\
\hline Hemicellulose $^{3}$ & 13.44 & 12.64 & 12.98 & 19.84 & 19.02 & 18.00 \\
\hline ADL & 1.42 & 1.42 & 1.40 & 3.94 & 3.83 & 3.53 \\
\hline Cellulose $^{4}$ & 4.55 & 4.93 & 4.40 & 7.86 & 8.36 & 9.03 \\
\hline
\end{tabular}

1 Concentrate mixture was composed of $60 \%$ yellow corn, $20 \%$, soy bean meal, $17 \%$ wheat bran, $0.8 \%$ sodium chloride $1.2 \%$ limestone and $1 \%$ common salt.

2 Fat source, Magnapac ${ }^{\circledR}$ is a commercial product of calcium salts of long chain fatty acids of palm oil. $\quad 3$ Hemicellulose $=\mathrm{NDF}-\mathrm{ADF} \quad 4$ Cellulose $=\mathrm{ADF}-\mathrm{ADL}$

\section{Rumen fluid parameters}

Rumen fluid samples were collected at the end of the metabolism trials from four lambs from each group, by using stomach tube. Samples were collected for two consecutive days before and $4 \mathrm{hrs}$ post feeding. Rumen fluid samples were filtered through four layers of cheese cloth. Ruminal $\mathrm{pH}$ was immediately determined using Orion Research digital pH meter, model (201). Ammonia-nitrogen (Conway, 1962), total VFA's concentrations (Kromann et al., 1967) and molar proportions of fatty acids (Erwin et al. 1961), total nitrogen and non- protein nitrogen (A.O.A.C., 1984) were determined. True protein nitrogen was calculated by the difference between total nitrogen and non- protein nitrogen.

\section{Blood plasma constituents}

Blood samples were collected from the same lambs at the end of metabolism trials from the left jugular vein in heparinized test tubes before and $4 \mathrm{hrs}$ post feeding. Blood plasma was spent by centrifugation of blood samples at $5.000 \mathrm{rpm}$ for 
15 minutes. Plasma were kept frozen at $-20{ }^{\circ} \mathrm{C}$ for total lipids, triglycerides and cholesterol analyses (Roche Diagnostics GmbH kits, D-68298, Mannheim).

Data collected were subjected to statistical analysis as two factor factorial analysis of variance (Mstat C., 1989). Duncan's Multiple Range Test (Duncan, 1955) was used to separate means when the dietary treatment effect was significant according to the following model 1.

$\mathrm{Y}_{\mathrm{ijk}}=\mathrm{M}+\mathrm{F}_{\mathrm{i}}+\mathrm{R}_{\mathrm{j}}+(\mathrm{FR})_{\mathrm{ij}}+\mathrm{e}_{\mathrm{ijk}} \quad$ (model 1)

Ruminal and blood plasma data according to model:

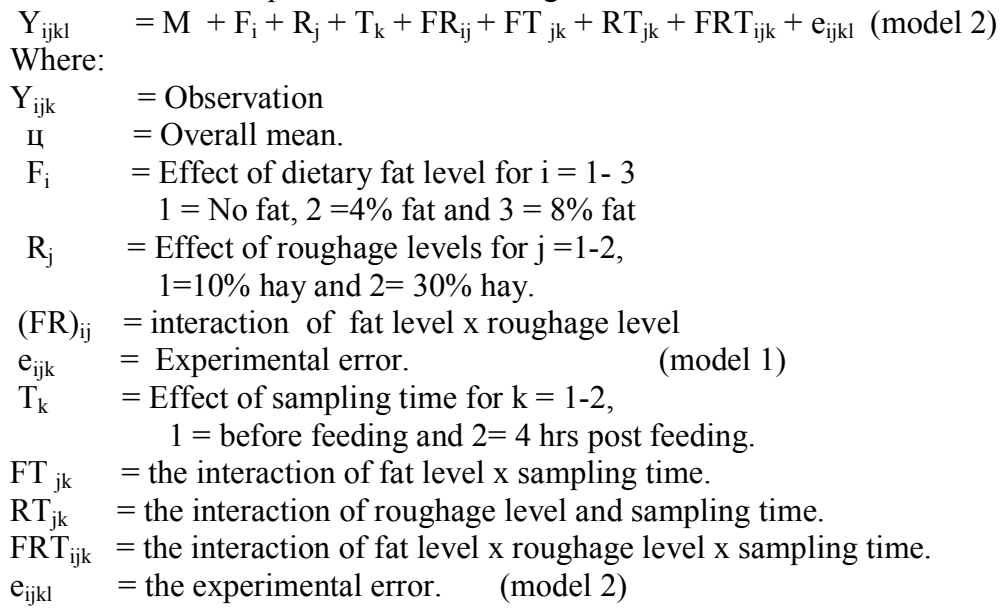

\section{RESULTS AND DISCUSSION}

Feed and chemical composition of the experimental rations are shown in Table 1. Protected fat was used to replace $4.2 \%$ of concentrate dry matter in $0 \%$ or $8 \%$ in high fat diets. Rations were almost iso-nitrogenous containing 19.38, 19.07 and $18.81 \%$ for low roughage diets and $19.09,18.75$ and $17.35 \%$ for high roughage one. The corresponding ether extract contents were 2.90, 6.37 and $9.84 \%$ for low roughage diet and $2.65,6.12$ and $9.59 \%$ for high roughage one. The NDF was about $19 \%$ for low roughage rations and $30 \%$ for high roughage level. The experimental rations differed in $\mathrm{EE}$ and $\mathrm{CF}$ content due to fat supplemented and roughage level (Table 1).

Inclusion of $4 \%$ protected fat did not affect DM digestibility but increasing fat level to $8 \%$ decreased $(\mathrm{P}<0.05) \mathrm{DM}$ digestibility by about 4 digestion units (Table 2). Canale et al. (1990) found that adding of $0.5 \mathrm{~kg}$ calcium soaps to dairy cow's ration increased the apparent digestibility of DM when diets contained $25 \% \mathrm{NDF}$. Hill and West (1991) found that DM digestibility was higher when $4.5 \%$ calcium soaps was added to the diet compared to the control with no added fat. However, ElBedawy et al. (2004b) found that protected fat supplement to growing-finishing bulls insignificantly $(\mathrm{P}>0.05)$ decreased the $\mathrm{DM}$ digestibility.

Digestibility of OM improved by incorporation of $4 \%$ dietary fat. This was not evident with $8 \%$ fat level. The same trend was observed for CP digestibility. ElBedawy et al. (2003) noticed that OM digestibility was significantly increased when calcium soaps was added at $4 \%$ and $8 \%$ levels to the rations. While El-Bedawy et al. 
(2004b) found that protected fat containing ration decreased OM digestibility and Zinn (1992) found no significant effect on total tract digestion of OM for beef cattle that fed $5 \%$ fat level.

The digestibility of CP was improved by incorporation of $4 \%$ dietary fat but this was not evident with the $8 \%$ fat level. Feeding $4 \%$ fat containing ration increased $\mathrm{CP}$ digestibility by $2.4 \%$ while, feeding $8 \%$ fat level decreased CP digestibility by $1.2 \%$ compared to control (no added fat). Palmquist and Conard (1978) and El- Bedawy et al. (2003) reported that addition of fat to the diet had no significant effect on digestibility of nitrogen. Jenkins and Palmquist (1984) found that added fatty acid in forms of Ca- Soap slightly increased nitrogen digestibility. However, El-Bedawy et al. (2004b) observed that protected fat supplemented diet at 5\% insignificantly $(\mathrm{P}>0.05)$ decreased $\mathrm{CP}$ digestibility.

Increasing dietary fat increased EE digestibility. No significant differences between $4 \%$ and $8 \%$ fat level in EE digestibility. The higher digestibility of EE associated with fat supplement in ration might be related to the higher digestibility of supplementary fat (Palmquist and Conrad, 1978). El-Bedawy (1995) found that supplemented fat either from oil or Ca- Soap significantly increased EE digestibility from $70 \%$ to $90 \%$, which could be due to the high digestibility of fatty acid in supplementary fat. Also, El-Bedawy et al. (2004a) found that EE digestibility increased by $19 \%$ when calcium soaps was added to the Frisian bull rations. However, El-Bedawy et al. (2004b) found that EE digestibility insignificantly $(\mathrm{P}>0.05)$ decreased by feeding protected fat containing diets to fattening bulls.

Crude fiber digestibility was not significantly influenced by increasing dietary fat level. However, NFE digestibility was decreased with $8 \%$ fat level. These results were in agreement with the findings of El- Bedawy (1995) in a study on sheep and El-Bedawy et al. (2004a) and El-Bedawy et al. (2004b) in a study on finishing bulls that calcium soap supplement had no significant effect on CF digestibility.

Roughage level had no significant effect on nutrient digestibility. However, slight decrease was observed for all nutrients by feeding high roughage level except for DM and CF, which showed slight increase.

Table 2. Main effects of dietary treatments on nutrient digestibilities of the experimental rations

\begin{tabular}{|c|c|c|c|c|c|c|c|}
\hline \multirow[t]{2}{*}{ Item } & \multicolumn{3}{|c|}{ Calcium soap, \% } & \multirow{2}{*}{ SEM } & \multicolumn{2}{|c|}{ Roughage, $\%$} & \multirow{2}{*}{ SEM } \\
\hline & 0 & 4 & 8 & & 10 & 30 & \\
\hline DM & $84.37^{\mathrm{a}}$ & $85.58^{\mathrm{a}}$ & $80.05^{b}$ & 1.09 & 82.63 & 84.04 & 1.10 \\
\hline $\mathrm{OM}$ & $74.69^{\mathrm{b}}$ & $77.25^{\mathrm{a}}$ & $73.80^{\mathrm{b}}$ & 0.83 & 76.54 & 73.95 & 0.69 \\
\hline $\mathrm{CP}$ & $70.66^{\mathrm{b}}$ & $72.33^{\mathrm{a}}$ & $69.78^{\mathrm{b}}$ & 0.97 & 72.41 & 69.78 & 0.72 \\
\hline $\mathrm{EE}$ & $45.77^{\mathrm{b}}$ & $82.78^{\mathrm{a}}$ & $84.86^{\mathrm{a}}$ & 1.61 & 74.06 & 68.22 & 5.47 \\
\hline $\mathrm{CF}$ & 40.28 & 43.97 & 43.97 & 1.88 & 40.81 & 44.67 & 1.48 \\
\hline NFE & $81.06^{\mathrm{a}}$ & $82.26^{\mathrm{a}}$ & $76.82^{\mathrm{b}}$ & 0.69 & 80.36 & 79.73 & 0.87 \\
\hline
\end{tabular}

${ }^{\mathrm{a}, \mathrm{b}}$ Means within each treatment having different superscripts $(\mathrm{P}<0.05)$ differ

Data in Table 3 showed the fat $\mathrm{x}$ roughage level interaction on nutrient digestibility. Feeding high fat level $8 \%$ with low roughage diet decreased DM digestibility (77.26). However this effect was not evident with high roughage diet $(82.85 \%)$. Addition of $4 \%$ fat containing diet slightly increased DM, OM, EE, CF, 
CP and NFE digestibilities. Zinn and Plascencia (1996) found that forage level did not affect $(\mathrm{P}>0.10)$ total tract digestion of fiber, starch and nitrogen. However, as excepted, increasing forage level decreased $(\mathrm{P}<0.01)$ total tract digestion of $\mathrm{OM}$. The same authors found no interaction $(\mathrm{P}>0.10)$ between forage level and supplemental fat on total tract digestibility of OM, lipid and starch.

Table 3. Effects of dietary fat and roughage level on nutrient digestibilities of the experimental rations

\begin{tabular}{lccccccc}
\hline \multirow{2}{*}{ Item } & \multicolumn{3}{c}{$10 \%$ roughage diets } & \multicolumn{3}{c}{$30 \%$ roughage diets } & \multirow{2}{*}{ SEM } \\
\cline { 2 - 6 } & \multicolumn{3}{c}{ Calcium soap, \% } & \multicolumn{3}{c}{ Calcium soap, \% } & \\
\cline { 2 - 6 } & 0 & 4 & 8 & 0 & 4 & 8 & 1.35 \\
DM & $83.79^{\mathrm{a}}$ & $86.84^{\mathrm{a}}$ & $77.26^{\mathrm{b}}$ & $84.96^{\mathrm{a}}$ & $84.32^{\mathrm{a}}$ & $82.85^{\mathrm{a}}$ & 1.90 \\
$\mathrm{OM}$ & $76.68^{\mathrm{ab}}$ & $78.50^{\mathrm{a}}$ & $74.44^{\mathrm{bcd}}$ & $72.71^{\mathrm{d}}$ & $76.00^{\mathrm{abc}}$ & $73.15^{\mathrm{cd}}$ & 0.90 \\
$\mathrm{CP}$ & $72.80^{\mathrm{a}}$ & $72.77^{\mathrm{a}}$ & $71.66^{\mathrm{ab}}$ & $68.52^{\mathrm{b}}$ & $71.90^{\mathrm{ab}}$ & $68.93^{\mathrm{ab}}$ & 1.15 \\
$\mathrm{EE}$ & $53.81^{\mathrm{b}}$ & $83.83^{\mathrm{a}}$ & $84.55^{\mathrm{a}}$ & $37.73^{\mathrm{c}}$ & $81.74^{\mathrm{a}}$ & $85.18^{\mathrm{a}}$ & 1.29 \\
$\mathrm{CF}$ & 39.09 & 42.48 & 40.87 & 41.47 & 45.47 & 47.08 & 2.43 \\
NFE & $81.74^{\mathrm{a}}$ & $82.76^{\mathrm{a}}$ & $76.58^{\mathrm{b}}$ & $80.38^{\mathrm{a}}$ & $81.77^{\mathrm{a}}$ & $77.06^{\mathrm{b}}$ & 0.91 \\
\hline a,b,c, d & \multicolumn{7}{c}{ Means in the same row having different superscripts $(\mathrm{P}<0.05)$ differ. }
\end{tabular}

Feeding 4\% fat containing diet improved $(\mathrm{P}<0.05)$ NDF, ADF, hemicellulose and cellulose digestibilities. However, feeding $8 \%$ fat level insignificant decreased $(\mathrm{P}>0.05)$ cellulose digestibility. High roughage level $(30 \%$ hay $)$ increased the digestibilities of cell wall constituents by $8 \%$ for NDF; $15 \%$ for ADF; $9 \%$ for hemicellulose and $11 \%$ for cellulose, respectively (Table 4).

The interactions between fat level and roughage level ( $\mathrm{F} \times \mathrm{R})$ was significant $(\mathrm{P}<0.01)$ for NDF, ADF, hemicellulose and cellulose digestibilities (Table 5). These results were in agreement with those obtained by Jenkins and Palmquist (1984), that the digestibility of ADF was found to be higher with added $4.5 \%$ calcium soap than the control. While Schauff and Clark (1989); Zinn (1992); Brinkmann and Abel (1992) and El-Bedawy et al. (2004a) found that apparent digestibilities of NDF and $\mathrm{ADF}$ were not affected by feeding fat containing rations.

Table 4. Main effects of dietary treatments on digestibility of cell wall constituents of the experimental ratios

\begin{tabular}{|c|c|c|c|c|c|c|c|}
\hline \multirow[t]{2}{*}{ Item } & \multicolumn{3}{|c|}{ Calcium soap, $\%$} & \multirow{2}{*}{ SEM } & \multicolumn{2}{|c|}{ Roughage, \% } & \multirow{2}{*}{ SEM } \\
\hline & 0 & 4 & 8 & & 10 & 30 & \\
\hline NDF & $60.13^{b}$ & $69.23^{\mathrm{a}}$ & $68.90^{\mathrm{a}}$ & 1.06 & $63.58^{b}$ & $68.60^{\mathrm{a}}$ & 1.33 \\
\hline $\mathrm{ADF}$ & $50.30^{\mathrm{b}}$ & $58.78^{\mathrm{a}}$ & $51.13^{\mathrm{b}}$ & 1.56 & $49.72^{b}$ & $57.07^{\mathrm{a}}$ & 1.35 \\
\hline Hemicellulose & $65.26^{\mathrm{c}}$ & $75.22^{b}$ & $78.55^{\mathrm{a}}$ & 1.30 & $69.95^{\mathrm{b}}$ & $76.07^{\mathrm{a}}$ & 1.76 \\
\hline Cellulose & $55.58^{\mathrm{b}}$ & $62.96^{\mathrm{a}}$ & $54.63^{\mathrm{b}}$ & 1.48 & $54.77^{b}$ & $60.68^{\mathrm{a}}$ & 1.31 \\
\hline
\end{tabular}

${ }^{\mathrm{a}, \mathrm{b}}$ Means within each treatment having different superscripts $(\mathrm{P}<0.05)$ differ.

Increasing fat level to $8 \%$ increased digestible energy (DE) and TDN but decreased the DCP content (Table 6). Andrew et al. (1991); Hill and West (1991) and El-Bedawy et al. (2004a) reported that inclusion of calcium soaps in rations increased digestible energy by 10 to $12 \%$. Zinn and Plascencia (1996) found that supplementary fat increased $(\mathrm{P}<0.01)$ the $\mathrm{DE}$ and $\mathrm{ME}$ values of the diet. However, 
El-Bedawy et al. (2004b) found that TDN values decreased for bulls fed fat containing rations. The DCP content of fat containing rations was lower than the control group. However, Bendary et al. (1994) found that DCP significantly increased by fat supplementation. High roughage rations were characterized by low digestible energy, TDN, CP and DCP values. Zinn and Plascencia (1996) found that increasing forage level decreased $(\mathrm{P}<0.01)$ dietary $\mathrm{DE}$ and $\mathrm{ME}$.

Table 5. Effects of dietary fat and roughage level on the digestibility of cell wall constituents of the experimental ratios

\begin{tabular}{lccccccc}
\hline & \multicolumn{9}{c}{$10 \%$ roughage diets } & \multicolumn{3}{c}{$30 \%$ roughage diets } & \\
\cline { 2 - 7 } Item & 0 & 4 & 8 & 0 & 4 & 8 & SEM \\
\hline NDF & $56.16^{\mathrm{d}}$ & $68.21^{\mathrm{b}}$ & $66.36^{\mathrm{b}}$ & $64.10^{\mathrm{c}}$ & $70.26^{\mathrm{a}}$ & $71.44^{\mathrm{a}}$ & 0.64 \\
ADF & $46.65^{\mathrm{c}}$ & $58.04^{\mathrm{a}}$ & $44.48^{\mathrm{c}}$ & $53.96^{\mathrm{b}}$ & $59.53^{\mathrm{a}}$ & $57.78^{\mathrm{a}}$ & 0.87 \\
Hemicellulose & $60.39^{\mathrm{e}}$ & $73.33^{\mathrm{c}}$ & $76.14^{\mathrm{b}}$ & $70.14^{\mathrm{d}}$ & $77.12^{\mathrm{b}}$ & $80.96^{\mathrm{a}}$ & 0.75 \\
Cellulose & $51.49^{\mathrm{c}}$ & $63.24^{\mathrm{a}}$ & $49.58^{\mathrm{c}}$ & $59.67^{\mathrm{b}}$ & $62.68^{\mathrm{ab}}$ & $59.68^{\mathrm{b}}$ & 1.10 \\
\hline
\end{tabular}

${ }^{\mathrm{a}, \mathrm{b}, \mathrm{c}, \mathrm{d}}$ Means in the same row having different superscripts $(\mathrm{P}<0.05)$ differ.

Table 6. Main effects of dietary treatments on nutritive value of the experimental rations

\begin{tabular}{|c|c|c|c|c|c|c|c|}
\hline \multirow[t]{2}{*}{ Item } & \multicolumn{3}{|c|}{ Calcium soap, $\%$} & \multirow{2}{*}{ SEM } & \multicolumn{2}{|c|}{ Roughage, \% } & \multirow{2}{*}{ SEM } \\
\hline & 0 & 4 & 8 & & 10 & 30 & \\
\hline DE Mcal / kg DM & $3.176^{b}$ & $3.460^{\mathrm{a}}$ & $3.554^{\mathrm{a}}$ & 0.05 & $3.487^{\mathrm{a}}$ & $3.306^{\mathrm{b}}$ & 0.06 \\
\hline TDN \% & $71.59^{\mathrm{b}}$ & $78.44^{\mathrm{a}}$ & $78.63^{\mathrm{a}}$ & 0.95 & $78.00^{\mathrm{a}}$ & $74.44^{\mathrm{b}}$ & 1.14 \\
\hline DCP $\%$ & $13.60^{\mathrm{a}}$ & $13.68^{\mathrm{a}}$ & $12.72^{\mathrm{b}}$ & 0.25 & $13.82^{\mathrm{a}}$ & $12.84^{\mathrm{b}}$ & 0.19 \\
\hline
\end{tabular}

${ }^{\mathrm{a}, \mathrm{b}}$ Means within each treatment having different superscripts $(\mathrm{P}<0.05)$ differ.

There were no interactions between fat level and roughage level (FxR) on TDN and DCP content (Table 7). These results are in agreement with those found by Zinn and Plascencia (1996).

Table 7. Effects of dietary fat and roughage level on nutritive value of the experimental rations

\begin{tabular}{|c|c|c|c|c|c|c|c|}
\hline \multirow[t]{2}{*}{ Item } & \multicolumn{3}{|c|}{$10 \%$ roughage diets } & \multicolumn{3}{|c|}{$30 \%$ roughage diets } & \multirow[t]{2}{*}{ SEM } \\
\hline & 0 & 4 & 8 & 0 & 4 & 8 & \\
\hline DE Mcal/ & $3.291^{\mathrm{c}}$ & $3.531^{\mathrm{ab}}$ & $3.638^{\mathrm{a}}$ & $3.061^{\mathrm{d}}$ & $3.338^{\mathrm{bc}}$ & $3.470^{\mathrm{ab}}$ & 0.05 \\
\hline TDN \% & $74.16^{\mathrm{c}}$ & $80.19^{\mathrm{a}}$ & $79.64^{\mathrm{ab}}$ & $69.01^{\mathrm{d}}$ & $76.68^{\mathrm{bc}}$ & $77.62^{\mathrm{ab}}$ & 0.88 \\
\hline DCP $\%$ & $14.11^{\mathrm{a}}$ & $13.88^{\mathrm{a}}$ & $13.48^{\mathrm{ab}}$ & $13.08^{\mathrm{b}}$ & $13.48^{\mathrm{ab}}$ & $11.96^{\mathrm{c}}$ & 0.22 \\
\hline
\end{tabular}

${ }^{\mathrm{a}, \mathrm{b}, \mathrm{c}, \mathrm{d}}$ Means in the same row having different superscripts differ $(\mathrm{P}<0.05)$.

Daily dry matter and nutrient intakes during the 70-day experimental period are shown in Table 8 . The mean values of concentrate intake by lambs fed high roughage level ranged from 754 to $762 \mathrm{~g} /$ day, while, it ranged from 874 to $912 \mathrm{~g} /$ day for lambs fed low roughage diets. On the other hand, roughage intake ranged from 275 to $285 \mathrm{~g}$ $/ \mathrm{h} /$ day for high roughage level but it ranged from 105 to $111 \mathrm{~g} / \mathrm{h} /$ day for lambs fed low roughage level.

Total dry matter intake (DMI) as $\mathrm{g} /$ day, $\mathrm{g} / \mathrm{kg} \mathrm{W} \mathrm{W}^{0.75}$ or $\mathrm{kg} / 100 \mathrm{~kg}$ BW were not significantly $(\mathrm{P}>0.05)$ different among the dietary treatments. These results might 
indicate that protected fat had no adverse effect on palatability. Comparable results were recorded for sheep by El-Bedawy (1989) and Ammann (1991). However, ElHag et al. (1985) and Ilian et al (1988) reported lower intake for fat supplemented sheep. On the other hand, Pantoja et al. (1996) and Elliott et al. (1996) reported higher intake when fat was added to the rations.

Digestible energy $(\mathrm{DE})$ and TDN intakes were higher $(\mathrm{P}<0.01)$ for protected fat supplemented group than the control (no added fat). The interaction between fat supplementation and roughage level $(\mathrm{F} \times \mathrm{R})$ on DE and TDN intakes was not significant. These results are in agreement with those found by El-Bedawy (1995) that TDN intake increased by fat addition in sheep diets.

No significant differences $(\mathrm{P}>0.05)$ in digestible crude protein (DCP) intake among the experimental groups were detected. Bendary et al. (1994); El-Bedawy et al. (1996) and El-Bedawy et al. (2004a) reported that fat addition had no significant effect on DCP intake.

Table 8. Dry matter, energy and nutrient intakes by the experimental groups

\begin{tabular}{|c|c|c|c|c|c|c|c|}
\hline \multirow[t]{2}{*}{ Item } & \multicolumn{3}{|c|}{$10 \%$ roughage diets } & \multicolumn{3}{|c|}{$30 \%$ roughage diets } & \multirow[t]{2}{*}{ SEM } \\
\hline & 0 & 4 & 8 & 0 & 4 & 8 & \\
\hline Av. body weight & 34.6 & 34.8 & 35.9 & 34.5 & 34.8 & 35.2 & 1.0 \\
\hline Concentrate mix. & 912 & 874 & 882 & 762 & 754 & 758 & 33 \\
\hline Berseem hay & 105 & 109 & 111 & 275 & 275 & 285 & 10 \\
\hline Protected fat & 0 & 43 & 87 & 0 & 45 & 90 & 2 \\
\hline \multicolumn{8}{|l|}{ Dry matter } \\
\hline g/day & 1017 & 1026 & 1080 & 1037 & 1074 & 1133 & 44.0 \\
\hline $\mathrm{g} / \mathrm{kg} \mathrm{W}^{0.75}$ & 71.30 & 71.61 & 73.67 & 72.80 & 74.90 & 78.48 & 3.06 \\
\hline $\mathrm{Kg} / 100 \mathrm{~kg} \mathrm{BW}$ & 2.94 & 2.95 & 3.01 & 3.00 & 3.08 & 3.22 & 0.13 \\
\hline \multicolumn{8}{|l|}{ DE (Mcal) } \\
\hline Mcal/ day & $2.93^{\mathrm{b}}$ & $2.99^{\mathrm{b}}$ & $3.52^{\mathrm{ab}}$ & $2.71^{\mathrm{b}}$ & $3.26^{\mathrm{ab}}$ & $3.90^{\mathrm{a}}$ & 0.21 \\
\hline Mcal/100 kg BW & $8.5^{\mathrm{b}}$ & $8.6^{\mathrm{b}}$ & $9.8^{\mathrm{ab}}$ & $7.9^{\mathrm{b}}$ & $9.4^{\mathrm{ab}}$ & $11.1^{\mathrm{a}}$ & 0.6 \\
\hline $\mathrm{Kcal} / \mathrm{W}^{0.75}$ & $205^{\mathrm{b}}$ & $209^{b}$ & $240^{\mathrm{ab}}$ & $190^{\mathrm{b}}$ & $227^{\mathrm{ab}}$ & $270^{\mathrm{a}}$ & 15 \\
\hline \multicolumn{8}{|l|}{ TDN } \\
\hline g/day & $755^{\mathrm{bc}}$ & $823^{\mathrm{ab}}$ & $860^{\mathrm{a}}$ & $716^{\mathrm{c}}$ & $823^{\mathrm{ab}}$ & $879^{a}$ & 33.5 \\
\hline $\mathrm{g} / \mathrm{kg} \mathrm{W}^{0.75}$ & $52.9^{b c}$ & $57.4^{\mathrm{abc}}$ & $58.7^{\mathrm{ab}}$ & $50.3^{\mathrm{c}}$ & $57.4^{\mathrm{abc}}$ & $60.9^{\mathrm{a}}$ & 2.30 \\
\hline $\mathrm{Kg} / 100 \mathrm{~kg} \mathrm{BW}$ & $2.18^{b c}$ & $2.37^{\mathrm{ab}}$ & $2.40^{\mathrm{ab}}$ & $2.07^{\mathrm{c}}$ & $2.36^{\mathrm{ab}}$ & $2.50^{\mathrm{a}}$ & 0.09 \\
\hline \multicolumn{8}{|l|}{ DCP } \\
\hline g/day & 143 & 142 & 146 & 136 & 145 & 136 & 6 \\
\hline $\mathrm{g} / \mathrm{kg} \mathrm{W}^{0.75}$ & 10.0 & 9.9 & 10.0 & 9.6 & 10.1 & 9.4 & 0.4 \\
\hline $\mathrm{g} / 100 \mathrm{~kg} \mathrm{BW}$ & 413 & 408 & 407 & 394 & 416 & 387 & 2 \\
\hline
\end{tabular}

$\overline{a, b, c}$ Means in the same row having different superscripts $(\mathrm{P}<0.05)$ differ.

Addition of fat at $8 \%$ level increased $(\mathrm{p}<0.05)$ water intake. However, no significant differences were found in water intake between the control and $4 \%$ fat group. Feeding high roughage diet increased $(\mathrm{p}<0.05)$ water intake by about $19 \%$ than low roughage level did (Table 9). However, El-Bedawy et al. (1996) reported lowest water intake by calves fed fat containing rations.

The increasing effect of roughage level on water intake was much pronounced with the high fat level (Table 10). Bartle et al. (1994) and Zinn and Plascencia (1996) 
found that increasing roughage level from $10 \%$ up to $30 \%$ in fat containing rations fed to steers $(\mathrm{P}<0.05)$ increased water consumption. The effect of dietary treatment on water intake was not related to change in dry matter intake which was almost constant but it was specific effect of roughage and fat levels.

Table 9. Main effects of dietary treatment on water intake by the experimental groups

\begin{tabular}{|c|c|c|c|c|c|c|c|}
\hline \multirow[t]{2}{*}{ Item } & \multicolumn{3}{|c|}{ Calcium soap, $\%$} & \multirow{2}{*}{ SEM } & \multicolumn{2}{|c|}{ Roughage, $\%$} & \multirow[t]{2}{*}{ SEM } \\
\hline & 0 & 4 & 8 & & 10 & 30 & \\
\hline $\mathrm{ml} /$ day & $2099^{b}$ & $1873^{b}$ & $2673^{a}$ & 142 & $2033^{b}$ & $2397^{\mathrm{a}}$ & 89 \\
\hline $\mathrm{ml} / \mathrm{kg} \mathrm{W}^{0.75}$ & $147^{\mathrm{b}}$ & $131^{\mathrm{b}}$ & $184^{\mathrm{a}}$ & 10 & $141^{\mathrm{b}}$ & $167^{\mathrm{a}}$ & 10 \\
\hline $\mathrm{ml} / 100 \mathrm{~kg} \mathrm{BW}$ & $6.1^{\mathrm{b}}$ & $5.4^{\mathrm{b}}$ & $7.5^{\mathrm{a}}$ & 0.4 & $5.8^{\mathrm{b}}$ & $6.9^{\mathrm{a}}$ & 0.4 \\
\hline $\mathrm{L} / \mathrm{kg} \mathrm{DM}$ intake & $2.04^{\mathrm{b}}$ & $1.78^{b}$ & $2.41^{\mathrm{a}}$ & 0.13 & 1.95 & 2.21 & 0.12 \\
\hline
\end{tabular}

${ }^{\mathrm{a}, \mathrm{b}}$ Means within each treatment having different superscripts $(\mathrm{P}<0.05)$ differ.

Table 10. Effects of dietary fat and roughage level on water intake by the experimental groups

\begin{tabular}{|c|c|c|c|c|c|c|c|}
\hline \multirow[t]{2}{*}{ Item } & \multicolumn{3}{|c|}{$10 \%$ roughage diets } & \multicolumn{3}{|c|}{$30 \%$ roughage diets } & \multirow[t]{2}{*}{ SEM } \\
\hline & 0 & 4 & 8 & 0 & 4 & 8 & \\
\hline $\mathrm{ml} / \mathrm{h}$ day & $1956^{\mathrm{bc}}$ & $1761^{\mathrm{c}}$ & $2381^{b}$ & $2241^{b c}$ & $1985^{\mathrm{bc}}$ & $2965^{\mathrm{a}}$ & 179 \\
\hline $\mathrm{ml} / \mathrm{kg} \mathrm{W}^{0.75}$ & $137^{\mathrm{b}}$ & $123^{\mathrm{b}}$ & $162^{b}$ & $157^{\mathrm{b}}$ & $138^{\mathrm{b}}$ & $205^{\mathrm{a}}$ & 13 \\
\hline $\mathrm{ml} / 100 \mathrm{~kg} \mathrm{BW}$ & $5.66^{\mathrm{b}}$ & $5.06^{\mathrm{b}}$ & $6.64^{\mathrm{b}}$ & $6.49^{b}$ & $5.70^{\mathrm{b}}$ & $8.44^{\mathrm{a}}$ & 0.51 \\
\hline $\mathrm{ml} / \mathrm{g} \mathrm{DM}$ intake & $1.9^{\mathrm{b}}$ & $1.7^{\mathrm{b}}$ & $2.2^{\mathrm{ab}}$ & $2.2^{\mathrm{ab}}$ & $1.9^{\mathrm{b}}$ & $2.6^{\mathrm{a}}$ & 0.2 \\
\hline
\end{tabular}

${ }^{\mathrm{a}, \mathrm{b}, \mathrm{c}}$ Means in the same row having different superscripts $(\mathrm{P}<0.05)$ differ.

Dietary treatments had no significant effect on nitrogen balance of the experimental groups (Table 11). Increasing fat to $8 \%$ insignificantly increased $(\mathrm{P}>0.05)$ nitrogen intake, fecal and urinary nitrogen loss as well as total nitrogen excretion. However, feeding $4 \%$ fat containing rations $(\mathrm{P}>0.05)$ improved nitrogen retention by $15 \%$ in comparison with control or $8 \%$ fat rations. Sklan (1989) found that inclusion of calcium soap to diet slightly improved nitrogen retention. Roughage level had no significant effect on nitrogen balance. Feeding high roughage ration $(\mathrm{P}>0.05)$ has no significant effect on nitrogen intake and output. Similar results were found by Zinn and Plascenica (1996) for steers.

Table 11. Main effects of dietary treatments on nitrogen balance (g/day) of the experimental groups

\begin{tabular}{lllllllll}
\hline \multirow{2}{*}{ Item } & \multicolumn{3}{l}{ Calcium soap, \% } & \multirow{2}{*}{ SEM } & \multicolumn{2}{l}{ Roughage, \% } & \multirow{2}{*}{ SEM } \\
\cline { 2 - 3 } & 0 & 4 & 8 & & 10 & 30 & \\
\hline Nitrogen intake & 27.35 & 27.57 & 30.69 & 1.38 & 27.85 & 29.06 & 1.24 \\
Fecal nitrogen & 8.03 & 7.62 & 9.10 & 0.50 & 7.72 & 8.78 & 0.38 \\
Digested nitrogen & 19.32 & 19.95 & 21.59 & 1.02 & 20.13 & 20.28 & 0.89 \\
Urinary nitrogen excretion & 8.63 & 7.66 & 10.98 & 0.86 & 9.48 & 8.70 & 0.81 \\
Total nitrogen excretion & 16.65 & 15.28 & 20.08 & 1.15 & 17.20 & 17.49 & 1.12 \\
Nitrogen retention & 10.70 & 12.25 & 10.61 & 0.90 & 10.65 & 11.58 & 0.74 \\
\hline
\end{tabular}


Data in Table (12) showed no significant differences $(\mathrm{P}>0.05)$ among the dietary treatments. High roughage - medium fat diet showed the highest nitrogen retention but high roughage - low fat showed the lowest one. Bayourthe et al. (1993) found that nitrogen retention was improved due to decrease in both fecal and urinary nitrogen excretion of fat fed sheep. In contrary, El-Hag et al. (1985) found that feeding fat containing diet tended to $(\mathrm{P}<0.05)$ decrease nitrogen intake and retention for sheep and goats.

Table 12. Nitrogen intake, excretion and retention (g/day) of the experimental groups

\begin{tabular}{lccccccc}
\hline Item & \multicolumn{3}{c}{$10 \%$ roughage diets } & \multicolumn{3}{c}{$30 \%$ roughage diets } & \multirow{2}{*}{ SEM } \\
\cline { 2 - 7 } & 0 & 4 & 8 & 0 & 4 & 8 & \\
\hline Nitrogen intake & 27.67 & 25.78 & 30.10 & 27.04 & 28.86 & 31.28 & 1.94 \\
Fecal nitrogen & 7.55 & 7.10 & 8.51 & 8.51 & 8.14 & 9.70 & 0.67 \\
Digested nitrogen & 20.12 & 18.68 & 21.59 & 18.53 & 20.72 & 21.58 & 1.38 \\
Urinary nitrogen excretion & 8.47 & 8.15 & 11.83 & 8.80 & 7.18 & 10.13 & 1.18 \\
Total nitrogen excretion & 16.02 & 15.25 & 20.34 & 17.31 & 15.32 & 19.83 & 1.61 \\
Nitrogen retention & 11.65 & 10.53 & 9.76 & 9.73 & 13.54 & 11.45 & 1.09 \\
\hline
\end{tabular}

Feeding fat had no significant effect on ruminal parameters (Table 13). Chalupa et al. (1986) found that calcium soaps did not alter the fermentation in the rumen because of its low insolubility in rumen. Increasing roughage level $(\mathrm{P}<0.05)$ increased ruminal $\mathrm{pH}$, molar proportions of acetate and actate: propionate ratio but decreased molar proportions of propionate, iso butyrate and valerate. Ruminal VFA's concentrations, butyrate and iso butyrate, were not affected by roughage level. Lower $\mathrm{pH}$ and higher total VFA's concentrations, acetate, butyrate, iso butyrate, valerate and iso valerate were determined at $4 \mathrm{hrs}$ post-feeding, but propionate and acetate: propionate ratio were not affected by sampling time (Table 13). El-Bedawy et al. (2004a) found that molar proportions of acetate; propionate; butyrate; iso- butyrate; valerate and iso-valerate were higher after feeding than those before feeding

There was no significant roughage $\mathrm{x}$ fat interaction on molar proportions of acetate and A: $\mathrm{P}$ ratio. However, the interactions on molar proportions of propionate; butyrate; iso- butyrate; valerate and iso- valerate were significant (Table 14). Zinn and Plascencia (1996) found that the interactions between forage and fat (F x R) on molar proportions of volatile fatty acids were not significant $(\mathrm{P}<0.10)$.

The mechanism of how lipids interfere ruminal fermentation is a complex model involving partitioning of lipid into microbial cell membrane, potency of the lipid to disrupt membrane and cellular function, physical attachment of microbial cells to plant surfaces expression and activity of microbial hydrolytic enzymes (Jenkins, 1993).

Increasing calcium soap level in sheep diet had no significant effect on nitrogen metabolism in the rumen (Table 15). These results are in agreement with those obtained by Doreau et al. (1993); Elliott et al. (1996); Belknap and Trenkle (1999) that calcium salts of fatty acids are inert in the rumen and does not alter rumen fermentation. Jenkins (1993) suggested that protein metabolism in the rumen is altered only when fat supplements interfere with fermentation. The decrease in ruminal ammonia nitrogen associated with feeding calcium soap containing rations 
could be related to the probable increase in ammonia nitrogen absorption by rumen epithelium as the ruminal $\mathrm{pH}$ was increased (Tamminga and Doreau, 1991).

Table 13. Main effects of dietary treatments on rumen fluid parameters of the experimental groups

\begin{tabular}{|c|c|c|c|c|c|c|c|c|c|c|}
\hline \multirow[t]{2}{*}{ Item } & \multicolumn{3}{|c|}{ Calcium soaps, $\%$} & \multicolumn{3}{|c|}{ SEM Roughage, $\%$} & SEM & \multicolumn{2}{|c|}{ Sampling time, hrs } & \multirow[t]{2}{*}{ SEM } \\
\hline & 0 & 4 & 8 & & 10 & 30 & & 0 & 4 & \\
\hline $\mathrm{pH}$ & 5.95 & 5.95 & 6.03 & 0.11 & $5.72^{b}$ & $6.23^{\mathrm{a}}$ & 0.06 & $6.25^{\mathrm{a}}$ & $5.70^{\mathrm{b}}$ & 0.06 \\
\hline VFA's $\%$ & 4.74 & 5.91 & 5.54 & 0.37 & 5.38 & 5.41 & 0.32 & $4.82^{\mathrm{b}}$ & $5.98^{\mathrm{a}}$ & 0.33 \\
\hline Acetate $\%$ & 59.08 & 58.84 & 59.82 & 1.81 & $53.72^{\mathrm{b}}$ & $64.78^{\mathrm{a}}$ & 0.90 & $56.73^{\mathrm{b}}$ & $61.76^{\mathrm{a}}$ & 1.37 \\
\hline Propionate \% & 24.03 & 25.18 & 27.12 & 1.59 & $31.30^{\mathrm{a}}$ & $19.59^{\mathrm{b}}$ & 0.48 & 24.0 & 81 & 1.29 \\
\hline Buty & 11.6 & 11. & 10.67 & 0.41 & 11.12 & 11.34 & 0.34 & $9.99^{b}$ & $12.47^{\mathrm{a}}$ & 0.23 \\
\hline so but & 1.22 & 1.02 & 1.08 & 0.07 & $1.27^{\mathrm{a}}$ & $0.95^{\mathrm{b}}$ & 0.05 & $0.96^{\mathrm{b}}$ & $1.26^{\mathrm{a}}$ & 0.05 \\
\hline Valer & 1.98 & 2.26 & 2.37 & 0.16 & $2.61^{\mathrm{a}}$ & $1.79^{\mathrm{b}}$ & 0.09 & $1.94^{\mathrm{b}}$ & $2.47^{\mathrm{a}}$ & 0.12 \\
\hline so valers & 1.52 & 1.51 & 1.50 & 0.08 & 1.55 & 1.47 & 0.05 & $1.29^{\mathrm{b}}$ & $1.72^{\mathrm{a}}$ & 0.05 \\
\hline $\mathrm{A}: \mathrm{P}$ ratio & 2.45 & 2.33 & 2.20 & 0.21 & $1.73^{\mathrm{b}}$ & $3.32^{\mathrm{a}}$ & 0.05 & 2.35 & 2.47 & 1.17 \\
\hline
\end{tabular}

${ }_{\mathrm{a}, \mathrm{b}}$ Means within each treatment having different superscripts $(\mathrm{P}<0.05)$ differ

Table 14. Effect of feeding experimental rations on rumen fluid parameters of the experimental groups

\begin{tabular}{|c|c|c|c|c|c|c|c|c|}
\hline \multirow{2}{*}{ Item } & & \multicolumn{3}{|c|}{$10 \%$ roughage diets } & \multicolumn{3}{|c|}{$30 \%$ roughage diets } & \multirow{2}{*}{ SEM } \\
\hline & & 0 & 4 & 8 & 0 & 4 & 8 & \\
\hline \multirow[t]{2}{*}{$\overline{\mathrm{pH}}$} & 0 & $6.06^{b}$ & $6.08^{b}$ & $6.17^{b}$ & $6.35^{\mathrm{a}}$ & $6.36^{\mathrm{a}}$ & $6.45^{\mathrm{a}}$ & \multirow[b]{2}{*}{0.05} \\
\hline & 4hrs & $5.32^{\mathrm{c}}$ & $5.31^{\mathrm{c}}$ & $5.36^{\mathrm{c}}$ & $6.05^{\mathrm{b}}$ & $6.07^{\mathrm{b}}$ & $6.12^{\mathrm{b}}$ & \\
\hline \multirow[t]{2}{*}{ VFA's. $\%$} & 0 & $4.38^{\mathrm{b}}$ & $5.45^{\mathrm{b}}$ & $4.73^{\mathrm{b}}$ & $4.48^{\mathrm{b}}$ & $5.45^{\mathrm{b}}$ & $4.43^{\mathrm{b}}$ & \multirow{2}{*}{0.58} \\
\hline & $4 \mathrm{hrs}$ & $4.50^{\mathrm{b}}$ & $7.65^{\mathrm{a}}$ & $5.60^{\mathrm{b}}$ & $5.60^{\mathrm{b}}$ & $5.08^{\mathrm{b}}$ & $7.43^{\mathrm{a}}$ & \\
\hline \multirow[t]{2}{*}{ Acetate $\%$} & 0 & $51.60^{\mathrm{e}}$ & $50.30^{\mathrm{e}}$ & $51.26^{\mathrm{e}}$ & $61.79^{\mathrm{abc}}$ & $62.77^{\mathrm{ab}}$ & $62.66^{\mathrm{ab}}$ & \multirow{2}{*}{1.91} \\
\hline & 4hrs & $56.36^{\text {cde }}$ & $54.84^{\mathrm{de}}$ & $57.93^{b c d}$ & $66.57^{\mathrm{a}}$ & $67.46^{\mathrm{a}}$ & $67.42^{\mathrm{a}}$ & \\
\hline \multicolumn{2}{|c|}{ Propionate $\% 0$} & $27.96^{\mathrm{c}}$ & $29.34^{\mathrm{c}}$ & $32.27^{\mathrm{b}}$ & $17.75^{\mathrm{g}}$ & $18.35^{\mathrm{fg}}$ & $18.77^{\mathrm{efg}}$ & \multirow[b]{2}{*}{0.66} \\
\hline & $4 \mathrm{hrs}$ & $30.13^{\mathrm{bc}}$ & $32.14^{\mathrm{b}}$ & $53.97^{\mathrm{a}}$ & $20.27^{\mathrm{def}}$ & $20.89^{\text {de }}$ & $21.49^{\mathrm{d}}$ & \\
\hline \multicolumn{2}{|c|}{ Butyrate $\% 0$} & $10.68^{c}$ & $9.97^{\mathrm{c}}$ & $9.32^{\mathrm{c}}$ & $9.70^{c}$ & $10.07^{\mathrm{c}}$ & $10.21^{\mathrm{c}}$ & \multirow{2}{*}{0.47} \\
\hline & $4 \mathrm{hrs}$ & $13.40^{\mathrm{a}}$ & $12.51^{\mathrm{a}}$ & $10.85^{\mathrm{bc}}$ & $12.92^{\mathrm{a}}$ & $12.87^{\mathrm{a}}$ & $12.30^{\mathrm{ab}}$ & \\
\hline \multicolumn{2}{|c|}{ IsoButyrate $\% 0$} & $1.02^{\mathrm{c}}$ & $0.99^{\mathrm{cd}}$ & $0.95^{\text {cde }}$ & $0.94^{\text {cde }}$ & $0.84^{\mathrm{e}}$ & $1.03^{\mathrm{c}}$ & \multirow{2}{*}{0.04} \\
\hline & 4hrs & $1.98^{\mathrm{a}}$ & $1.40^{\mathrm{b}}$ & $1.28^{\mathrm{b}}$ & $0.96^{\text {cde }}$ & $0.86^{\mathrm{de}}$ & $1.05^{\mathrm{c}}$ & \\
\hline \multirow[t]{2}{*}{ Valerate $\%$} & 0 & $1.96^{\mathrm{cd}}$ & $2.10^{\mathrm{bcd}}$ & $2.26^{\mathrm{bcd}}$ & $1.69^{\mathrm{d}}$ & $1.73^{\mathrm{d}}$ & $1.93^{\mathrm{cd}}$ & \multirow{2}{*}{0.11} \\
\hline & $4 \mathrm{hrs}$ & $2.48^{\mathrm{b}}$ & $3.44^{\mathrm{a}}$ & $3.46^{\mathrm{a}}$ & $1.82^{\mathrm{cd}}$ & $1.76^{\mathrm{d}}$ & $1.85^{\mathrm{cd}}$ & \\
\hline \multicolumn{2}{|c|}{ Isovalerate \% } & $1.14^{\mathrm{d}}$ & $1.21^{\mathrm{d}}$ & $1.06^{\mathrm{d}}$ & $1.41^{\mathrm{c}}$ & $1.43^{\mathrm{c}}$ & $1.51^{\mathrm{c}}$ & \multirow{2}{*}{004} \\
\hline \multirow{3}{*}{$\mathrm{A}: \mathrm{P}$ ratio } & 4hrs & $2.09^{\mathrm{a}}$ & $1.96^{\mathrm{ab}}$ & $1.68^{\mathrm{b}}$ & $1.44^{\mathrm{c}}$ & $1.46^{\mathrm{c}}$ & $1.56^{\mathrm{c}}$ & \\
\hline & 0 & $1.85^{\mathrm{c}}$ & $1.71^{\mathrm{c}}$ & $1.59^{\mathrm{c}}$ & $3.48^{\mathrm{a}}$ & $3.42^{\mathrm{ab}}$ & $3.34^{\mathrm{ab}}$ & \multirow[b]{2}{*}{0.09} \\
\hline & 4hrs & $1.87^{\mathrm{c}}$ & $1.71^{\mathrm{c}}$ & $1.61^{\mathrm{c}}$ & $3.28^{\mathrm{ab}}$ & $3.23^{\mathrm{ab}}$ & $3.14^{\mathrm{b}}$ & \\
\hline
\end{tabular}

Increasing roughage level $(\mathrm{P}<0.05)$ decreased ruminal total nitrogen, non protein nitrogen, ammonia nitrogen and true protein nitrogen. Elliott et al. (1995); Bateman et al. (1996) and Zinn and Plascencia (1996) found that feeding high roughage rations insignificantly decreased $(\mathrm{P}>0.05)$ ruminal total nitrogen, ammonia nitrogen 
and true protein nitrogen. All forms of ruminal nitrogen were $(\mathrm{P}<0.05)$ lower at $4 \mathrm{hrs}$ post feeding than those before feeding

Data of Table 16 showed that there were no interactions $(p>0.05)$ between roughage level and supplemental fat $(\mathrm{F} \times \mathrm{R})$ on ruminal total nitrogen; non-protein nitrogen; ammonia nitrogen and true protein nitrogen. These results are in agreement with those found by Zinn and Plascencia (1996) that there were no interactions $(\mathrm{P}>0.10)$ between forage level and supplemental fat $(\mathrm{F} \times \mathrm{R})$ on ruminal nitrogen.

Table 15. Mean effects of dietary treatments on ruminal nitrogen fraction (mg/100ml) of the experimental groups

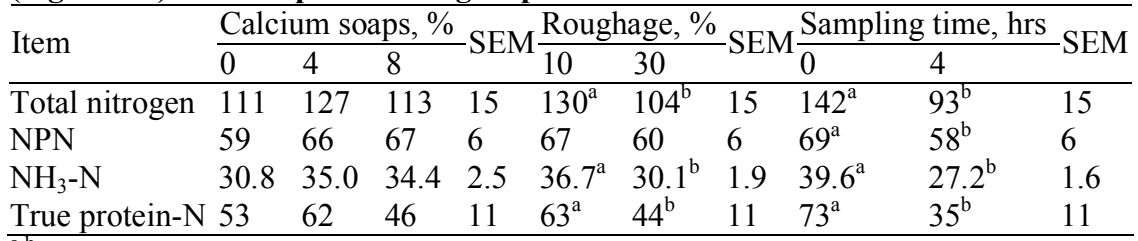

${ }^{a, b}$ Means within each treatment having different superscripts $(\mathrm{P}<0.05)$ differ

Table 16. Ruminal nitrogen fraction $(\mathrm{mg} / 100 \mathrm{ml})$ before and $4 \mathrm{hrs}$ post feeding for different experimental groups

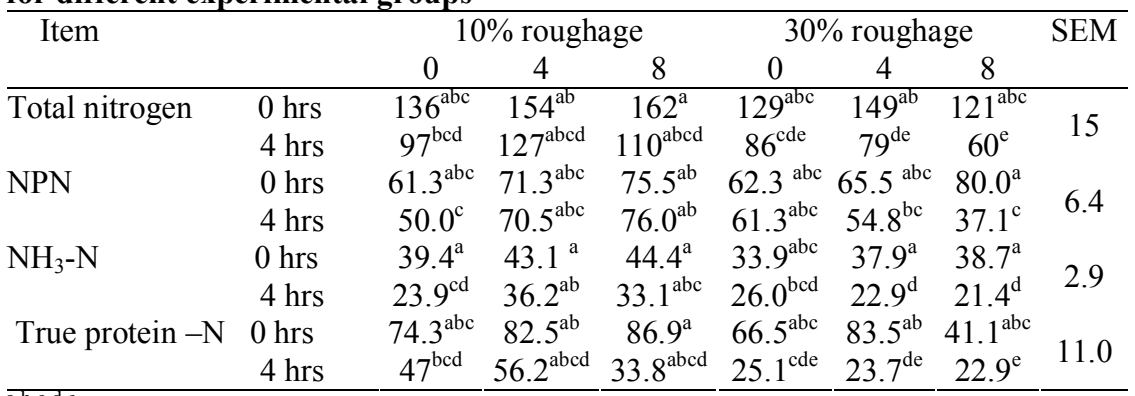

$\overline{\mathrm{a}, \mathrm{b}, \mathrm{c}, \mathrm{d}, \mathrm{e}}$ Means in the same row having different superscripts $(\mathrm{P}<0.05)$ differ.

Increasing protected fat level to $4 \%$ or $8 \%$ of dietary ration dry matter $(\mathrm{P}<0.05)$ increased total lipids and $(\mathrm{P}>0.05)$ triglycerides in blood plasma. Total cholesterol was $(\mathrm{P}<0.05)$ higher for $8 \%$ fat group than the control and $4 \%$ fat group (Table 17). On the other hand, increasing roughage level $(\mathrm{P}<0.05)$ increased total plasma lipids and total cholesterol but had no significant effect on triglycerides. Total cholesterol $(\mathrm{P}<0.05)$, total lipids and triglycerides $(\mathrm{P}>0.05)$ were higher at 4 hrs post feeding than those before feeding (Table 17). The increase in plasma total lipids of fat supplemented groups may be attributed to greater quantity of fatty acids absorbed from fat supplemented diets through the gut (Jenkins et al., 1989) and/or the fact that feeding fat is associated with depression in lipogenic enzyme activities by liver and adipose tissues (Steele, 1980 and Storry, 1981). Moreover, feeding long - chain fatty acids induced shifting in the balance from active polymeric to inactive polymeric forms of acetyl Co- A carboxyl's in bovine adipose tissues (Bauman and Davis, 1975). 
Table 17. Main effect of dietary treatments on blood plasma lipids $(\mathrm{mg} / 100 \mathrm{ml})$ of the experimental groups

\begin{tabular}{|c|c|c|c|c|c|c|c|c|c|c|}
\hline \multirow[t]{2}{*}{ Item } & \multicolumn{3}{|c|}{ Calcium soaps, $\%$} & & \multicolumn{2}{|c|}{ Roughage, \% } & \multirow{2}{*}{ SEM } & \multicolumn{3}{|c|}{ Sampling time, hrs $\mathrm{SEM}$} \\
\hline & 0 & 4 & 8 & & 10 & 30 & & 0 & 4 & \\
\hline t & $6.44^{\mathrm{b}}$ & $6.67^{\mathrm{a}}$ & $6.77^{\mathrm{a}}$ & 0.06 & $6.54^{b}$ & $6.71^{\mathrm{a}}$ & 0.05 & 6.60 & 6.65 & 0.05 \\
\hline Triglycerides & 89 & 94 & 100 & 5 & 94 & 95 & 4 & 89 & 99 & 4 \\
\hline Total cholesterol & $110^{b}$ & $114^{\mathrm{b}}$ & $141^{\mathrm{a}}$ & 8 & $110^{\mathrm{b}}$ & $133^{\mathrm{a}}$ & 7 & $107^{\mathrm{b}}$ & $137^{\mathrm{a}}$ & 7 \\
\hline
\end{tabular}

${ }^{\mathrm{a}, \mathrm{b}}$ Means within each treatment having different superscripts $(\mathrm{P}<0.05)$ differ.

No significant interaction was found between supplemental fat level and roughage level $(\mathrm{F} \times \mathrm{R})$ on total lipids and triglycerides. However, there were significant interactions $(\mathrm{F} \times \mathrm{R})$ on total cholesterol. Lambs fed high roughage- high fat diet showed the highest value of total lipids $(6.88 \mathrm{~g} / 100 \mathrm{ml})$ and total cholesterol $(205.2$ $\mathrm{mg} / 100 \mathrm{ml}$ ). Hill and West (1991) suggested many factors may influence plasma fatty acids concentrations including metabolism of other nutrients, level or composition of dietary fat, and production stage and type of production (meat or milk).

Table 18. Blood plasma lipids $(\mathrm{mg} / 100 \mathrm{ml})$ of the experimental groups

\begin{tabular}{|c|c|c|c|c|c|c|c|c|}
\hline \multirow[t]{2}{*}{ Item } & & \multicolumn{3}{|c|}{$10 \%$ roughage diets } & \multicolumn{3}{|c|}{$30 \%$ roughage diets } & \multirow{2}{*}{ SEM } \\
\hline & & 0 & 4 & 8 & 0 & 4 & 8 & \\
\hline \multirow[t]{2}{*}{ Total lipids } & $0 \mathrm{hrs}$ & $6.35^{\mathrm{d}}$ & $6.55^{\mathrm{abcd}}$ & $6.64^{\mathrm{abcd}}$ & $6.46^{\mathrm{bcd}}$ & $6.73^{\mathrm{abc}}$ & $6.86^{\mathrm{a}}$ & \multirow{2}{*}{0.1} \\
\hline & $4 \mathrm{hrs}$ & $6.40^{\mathrm{cd}}$ & $6.60^{\mathrm{abcd}}$ & $6.69^{\mathrm{abcd}}$ & $6.56^{\mathrm{abcd}}$ & $6.79^{\mathrm{ab}}$ & $6.88^{\mathrm{a}}$ & \\
\hline \multirow[t]{2}{*}{ Triglycerides } & $0 \mathrm{hrs}$ & $105.4^{\mathrm{ab}}$ & $88.8^{\mathrm{abc}}$ & $90.7^{\mathrm{abc}}$ & $73.4^{\mathrm{c}}$ & $86.8^{\mathrm{abc}}$ & $89.0^{\mathrm{abc}}$ & \multirow[b]{2}{*}{7.6} \\
\hline & $4 \mathrm{hrs}$ & $78.4^{\mathrm{bc}}$ & $86.3^{\mathrm{abc}}$ & $112.3^{\mathrm{a}}$ & $97.0^{\mathrm{abc}}$ & $114.2^{\mathrm{a}}$ & $108.2^{\mathrm{ab}}$ & \\
\hline \multicolumn{2}{|c|}{ Total cholesterol $0 \mathrm{hrs}$} & $115.7^{\mathrm{bcd}}$ & $90.9^{\mathrm{d}}$ & $102.3^{\mathrm{cd}}$ & $84.2^{\mathrm{d}}$ & $107.9^{\mathrm{bcd}}$ & $137.9^{\mathrm{bc}}$ & \multirow{2}{*}{11.2} \\
\hline & $4 \mathrm{hrs}$ & $119.9^{\mathrm{bcd}}$ & $111.3^{\mathrm{bcd}}$ & $118.2^{\mathrm{bcd}}$ & $118.9^{\text {bcd }}$ & $145.3^{\mathrm{b}}$ & $205.2^{\mathrm{a}}$ & \\
\hline
\end{tabular}

$\overline{a, b, c, d, e}$ Means in the same row having different superscripts $(\mathrm{P}<0.05)$ differ.

\section{Implications:}

The effect of adding protected fat to fattening diets of lambs was limited, however, incorporation of $4 \%$ calcium soap improved feed utilization of the $30 \%$ roughage diet to be comparable to that containing $90 \%$ concentrate without adverse effects on rumen metabolism and blood plasma metabolites.

\section{REFERENCES}

A.O.A.C., 1984. Official Methods of Analysis. $14^{\text {th }}$ Ed. Association of Official Analytical Chemists, Washington, D.C.

Ammann, HM., 1991. Influence of protected fat and protected protein given separately or together on physiology of digestion in the rumen, abomasum and feces of sheep. Thesis, Tierarztliche, Hochschule Hannover, Germany 176 pp. or (Nut. Abst. and Review 1992, S.B, 62: 4992.

Andrew, S.M., H.F. Tyrrell, C.K. Reynolds and R.A. Erdmen.,1991. Net energy for lactation of calcium salts of long- chain fatty acids for low fed silage- based diets. J. Dairy Sci., 74: 2588. 
Bartle, S.J., R.L. Preston and M.F. Miller., 1994. Dietary energy source and density: Effect of roughage source, roughage equivalent, tallow level and steer type on feedlot performance and carcass characteristics. J. Anim. Sci., 72:1943.

Bateman, H.G., J.N. Spain and M.R. Ellersieck., 1996. Influence of by- product feeds and tallow on lactation performance of Holstein cows during two seasons. J. Dairy Sci., 79: 114.

Bauman, D.E. and C.L. Davis, 1975. Regulation of lipid metabolism. In digestion and metabolism in the ruminant. (Ed. I.W. Mc Donald and A.C.I. Warner) PP 496. Armidale- Australia.

Bayourthe, C., R. Moncoulon and M. Vernay, 1993. Effect of protein- protected fat on ruminal and total nutrient digestibilities of sheep diets. J. Anim. Sci., 71: 1026.

Belknap, C. and A. Trenkle, 1999. Effect of feeding high- oil corn and control corn with added fat on ruminal and total tract digestion of finishing steers. J. Anim. Sci., 77, suppl., 1 No., 588 page 256 (Abstract).

Bendary, M.M., I.A. Abou- Selim, M.R.M. Moustafa, A.M Mahmoud and A.E.M. Khinizy, 1994. Performance of fattening buffalo calves fed different levels of fat for two different periods. Egyp. J.Anim. Prod. 31. suppl Issue: 613.

Brinkmann, J. and H.Abel, 1992. Effects of Ca- Saponified, esterified and free palm oil fatty acids on the development of rumen microbial population in continuous cultures and on nutrient digestibility and feed particle passage in sheep. J.Anim. Physiology and Anim. Nutr. 68: 3, 174.

Broudiscou, L., C. J. Van Nevel, D. I. Demeyer, and J. P. Jouany, 1988. Addition d'hydrolysat dhuile de soja dans la ration de mouton. Effet sur la degradation in sacco de la paille et de la cellulose. Reprod. Nutr. Dev. 28:159.

Canale, C.J., P.L. Burgess, L.D. Muller and G.V. Varga, 1990. Calcium salts of fatty acids in diets that differ in neutral detergent fiber. Effect on lactation performance and nutrient digestibilities. J.Dairy Sci., 73: 1439.

Chalupa, W., B. Rickabaugh, D. S. Kronfeld, and D. Sklan, 1984. Rumen fermentation in vitro as influenced by long chain fatty acids. J. Dairy Sci. 67:1439.

Chalupa, W., B.Vecchiarelli, A.E. Elser and D.S. Kronfeld, 1986. Ruminal fermentation in vivo as influenced by long chain fatty acids. J.Dairy Sci, 69: 1293.

Conway, E.J., 1962. Microdiffusion Analysis and Volumetric Error. London, Crosby Lockwood and Son.

Corine Bayourthe, R. Moncoulon, and Michhle Vernay, 1993. Effect of proteinprotected fat on ruminal and total nutrient digestibility of sheep diets. J. Anim. Sci. 71:1026.

Doreau, M., A. Ferlay and Y. El-Meddah, 1993. Organic matter and nitrogen digestion by dairy cows fed calcium salts of rapeseed oil fatty acids or rapeseed oil. J. Anim. Sci., 71:499.

Duncan, D.B., 1955. Multiple range and multiple F-test. J. Biometrics. 11:1.

El-Bedawy, T.M., 1989. Fat in small ruminant nutrition, preliminary study. 1: Effect of fat inclusion on intake, digestibility and growth performance of goats and sheep fed high concentrate diets. Third Egyptian British Conference on Animal, Fish and Poultry Production, Alex., 7- 10 October, Page: 393.

El-Bedawy, T.M., 1995. Preparation of sunflower oil calcium soap as a protected fat and its use in ruminant nutrition. J. Agric. Sci., Mansoura University, 20: 231. 
El-Bedawy, T.M., Sabbah, M. Allam, A.F. El- Kholy and A.K. Basiony, 1996. Response of growing buffalo calves to fat containing rations. Egyp. J. Anim. Prod., 33 : 79.

El-Bedawy, T.M., Sabbah, M. Allam, I.A. Gomaa and F.M. Omer, 2003. Effect of calcium soap on nutrient utilization, plasma lipids and performance of growingfinishing Friesian bulls.

El-Bedawy, T.M., I. A. Gommaa, Sabbah M. Allam and F.M. Abo-Donia, 2004a. Production of calcium salts of fatty acid from soap-stock on semi industrial scale and its use in finishing rations of Friesian bulls. Egypt. J. Anim. Prod. 41:19.

El-Bedawy, T.M., M.A.I. Salem and A.S. Sami, 2004b. Calcium soaps in low or high roughage rations: 1- Effect on digestion, rumen metabolism, blood plasma lipids of growing-finishing bulls. Egypt. J Anim. Prod. 41:31.

El-Hag, M.G., O.I. Kurdi and S.O. Mahgoup, 1985. Performance and carcass characteristics of Suddan desert sheep and goats on high roughage diets with added fat. Anim. Feed Sci., and Tech., 13: 147.

Elliott, J.P., J.K. Drackley and D.J. Weigel, 1996. Digestibility and effect of hydrogenated palm fatty acid distillate in lactating dairy cows. J. Dairy Sci., 79: 1031.

Elliott, J.P., J.K. Drackley, G.C. Fahey, Jr., and R.D. Shanks, 1995. Utilization of supplemental fat by dairy cows fed diets varying in content of nonstructural carbohydrates. J.Dairy Sci., 78: 1512.

Erwin, E.S., G.J. Marco and E.M. Emery, 1961. Volatile fatty acids analysis of blood and rumen fluid by gas chromatography. J. Dairy Sci. 44:1768.

Goering, H.K. and P.J. Van Soest, 1970. Forage fiber analyses (Apparatus, Reagents, Procedures and some applications). USDA, Agr. Hand. Book, 379.

Grummer, R. R., 1988. Influence of prilled fat and calcium salt of palm oil fatty acids on ruminal fermentation and nutrient digestibility. J. Dairy Sci. 71:117.

Hill, G.M. and J.W. West, 1991. Rumen protected fat in kline barley or corn diets for beef cattle: Digestibility, physiological, and feedlot responses. J. Anim. Sci. 69: 3376.

Ilian, MA., MA. Al- Awadi and AJ. Salman, 1988. Use of fat in diets of sheep in hot environments.1: Effects on performance, carcass characteristics and lipid composition of plasma. Anim. Feed Sci. and Technology, 19: 327.

Jenkins, T. C. and D. 1. Palmquist, 1984. Effect of fatty acids or calcium soaps on rumen and total nutrient digestibility of dairy rations. J. Dairy Sci., 67: 978.

Jenkins, T.C., 1993. Lipid metabolism in the rumen. J. Dairy Sci., 76: 3851.

Jenkins, T.C., T. Gimenez and D.L. Cross, 1989. Influence of phospholipids on ruminal fermentation in vitro and on nutrient digestion and serum lipid in sheep. J. Anim. Sci., 67: 529.

Kromann, R.P., J.H. Meyer and M.J. Stielu, 1967. Steam distillation of volatile fatty acids in rumen ingesta. J. Dairy Sci. 50:73.

Mstat C., 1989. Statistical package. Department of Crop and Soil Science. E. Lansing, Michigan 48824. USA.

Ostergaard, V., A. Danfaer, J. Dangaard, J. Hindhede, and I. Thysen, 1981. The effect of dietary lipids on milk production in dairy cows. p 508. Beretning Fra Statens Husdyrbrugs Forsog, Copenhagen, Denmark.

Palmquist, D. L., and T. C. Jenkins, 1980. Fat in lactation rations: review. J. Dairy Sci. 63:1. 
Palmquist, D.L. and H.R. Conrad, 1978. High fat rations for dairy cows: Effects on feed intake, milk and fat production and fat metabolites. J. Dairy Sci. 61:890

Pantoja, J., J.L. Firkins, M.L. Eastridge and B.L. Hull, 1996. Fatty acid digestion in lactating dairy cows fed fats varying in degree of saturation and different fiber sources. J. Dairy Sci., 79: 575.

Schauff, D.J. and J.H. Clark, 1989. Effects of prilled fatty acids and calcium salts of fatty acids on rumen fermentation, nutrient digestbilities, milk production and milk composition. J. Dairy Sci., 72: 917.

Sklan, D., 1989. In vitro and in vivo rumen protection proteins coated with calcium soaps of long- chain fatty acids. J. Agric. Sci. (Camb.) 112: 79.

Steele, W., 1980. The effects of soybean oil and type of forage in the diet on the plasma lipid composition of sheep. Br.J. Nutr. 44: 333.

Storry, J.E., 1981. The effect of dietary fat on milk composition. In Recent Advances in Animal Nutrition (W. Haresign, Ed.) PP. 3- 33.

Sutton, J. D., R. Knight, A. B. McAllan, and R. H. Smith, 1983. Digestion and synthesis in the rumen of sheep given diets supplemented with free or protected oils. Br. J. Nutr. 49: 419.

Tamminga, S. and M. Doreau, 1991. Lipids and rumen digestion. In: J.P. Jouany (Ed). Rumen microbial metabolism and ruminant digestion PP. 151. INRA, Paris, France.

Zinn, R.A., 1992. Comparative feeding value of supplemental fat in steam- flaked wheat based finishing diets for feedlot steers. J. Anim. Sci., 70: 2959.

Zinn, R.A. and A. Plascencia, 1996. Effects of forage level on the comparative feeding value of supplemental fat in growing- finishing diets for feedlot cattle. J. Anim. Sci., 74: 1194. 


\section{تأثير مستوى الاهن المحمى و المادة الخشنة على الهضم و التمثيل الغذائى فى الكرش

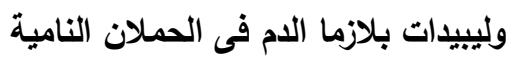

طه محمد البداوى'، سوسن منصور أحمد'، محمد على إبراهيم سالم' و حامد عبد العزيز على عمرَ'

اقسم الإنتاج الحيوانى، كلية الزراعة، جامعة القاهرة، الجيزة، مصر، باقسم الإنتاج الحيوانى، المركز القومى

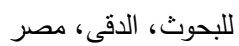

قسم سنون حملا من سلالة البرقى عمرها 1 شهور و منوسط أوزانها YV.O كجم عشوائيا إلى ست

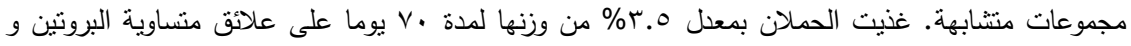

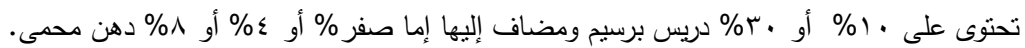

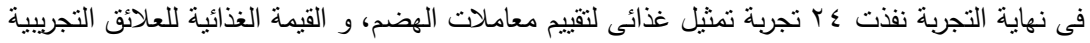

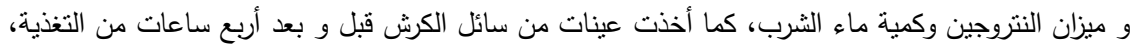

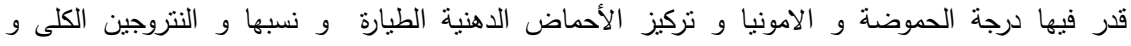
النتروجين غير البروتيني. وقدرت نسبة الليبيدات الكلية و الجلسريدات الثلاثية و الكوليسترول فى البلازما.

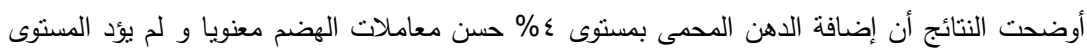

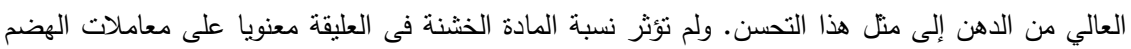
فيما عدا مكونات الجدار الخلوي التى زاد معامل هضمها فى العلائق العالية فى المادة الخشنة.

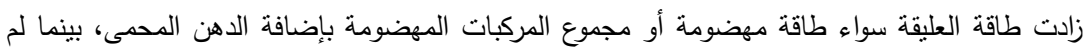
تتأثر نسبة البروتين الخام المهضوم بالمعاملات الغذائية.

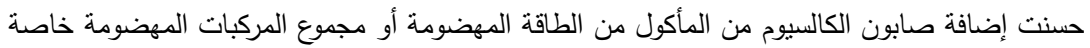

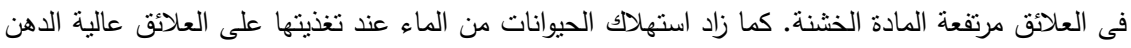
و المادة الخشنة، بينما لم يتأثز ميزان النتروجين بالمعاملات الغذائية.

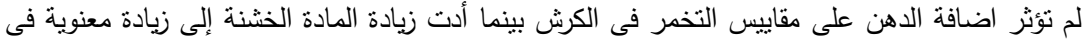

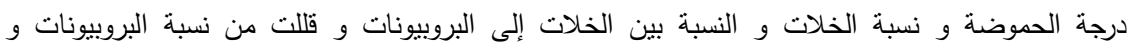

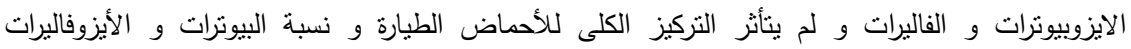

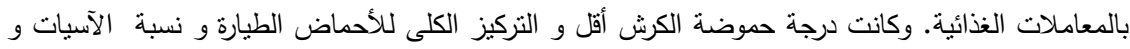

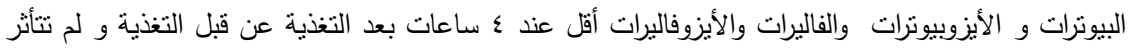

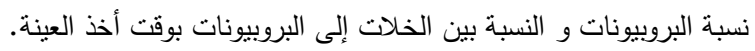

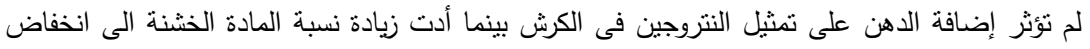
معنوى فى نسبة النتروجين الكلى ونسبة النتروجين غير البروتينى و نتروجين الامونيا و البروتين الحقيقى و لم لم تؤثر على نسبة النتروجين غير البروتينى فى الكرش. 
أدت إضافة الدهن إلي زيادة معنوية فى الليبيدات الكلية و الجلسريدات الثلاثية فى بلازما الدم، بينما زاد الكوليسترول معنويا فقط عند تغذية العلائق المحتوية على ^ ٪ \% دهن. و أدت زيادة نسبة المادة الخشنة إلي زيادة معنوية فى الليبيدات الكلية و الكوليسترول بينما لم نتأثر نسبة الجلسريدات الثنلاثية. وكانت قيم الكوليسترول بعد ؛ ساعات من بدء التغذية أعلى بدرجة معنوية و الليبيدات الكلية الجلسريدات الثلاثية بدرجة غير معنوية عن نفس القيم قبل التغذية.

إضافة §\% دهن محمى حسن الاستفادة الغذائية للعلائق المحتوية على نسبة عالية من المادة الخشنة

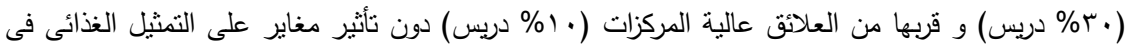

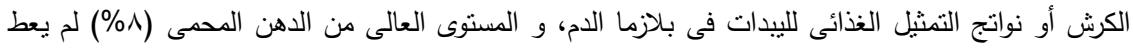
نتائج أفضل من هذا المسنوى. 